\title{
What Should Be Done if There Is Coronary Artery Disorder in Ruptured Abdominal Aortic Aneurysm?
}

\author{
Erdal Simsek, Mehmet Bayraktaroglu, Huseyin Bayram, Sevket Atasoy, Salih Fehmi Katircioglu \\ Departman of Cardiovascular Surgery, Ankara Etlik İhtisas Training and Research Hospital, Ankara, Turkey \\ E-mail:erdaldr@yahoo.com,mehmetbayraktaroglu@hotmail.com,drhuseyinbayram@gmail.com, \\ sevketatasoy@yahoo.com,ulusalbilim@gmail.com \\ Received August 5, 2011; revised August 27, 2011; accepted September 4, 2011
}

\begin{abstract}
Abdominal aortic aneurysm (AAA) is the most common type of aneurismal diseases. Generally, it is asymptomatic and when it is ruptured, it develops with high morbidity and mortality. Case report: A 62-years-old male patient consulted our emergency with a pain at his dorsum and lumbar part. Cardiologist with a suspicion of coronary artery disorder or dissection, coronary angiography was executed. Consecutive lesions of LAD artery (left anterior descending) 40\% - 50\% and 90\%, CX artery (circumflex) $40 \%$ and $80 \%$ - 90\%, and a lesion of RCA (right coronary artery) $20 \%$ - 30\% were detected. With a suspicion of rupture, abdominal aneurysm tomography (CT) was demanded. In the tomography, a 7-cm-diameter ruptured abdominal aortic aneurysm was diagnosed. Levosimendan support was started. Under the support of levosimendan a Y graft operation was performed. The operation was ended up with levosimendan support considering that coronary bypass would increase mortality and morbidity. Discussion: Approximately $50 \%$ of the ruptured aneurysms are died before they reach hospital while the $30 \%$ - $70 \%$ operated ones are died within 30 days after operation. Early diagnosis and follow-up is extremely important to decrease morbidity and mortality. The patients consulting with rupture must be taken to the operation without delay. What should be done if coronary artery disorder is detected in the patient whose AAA is ruptured and if the bypass is necessary? In our opinion, a decision must be made according to the patient's clinical condition. As a result of our case, we thought repairing the abdominal aortic aneurysm necessitates the other comorbidites must be treated medically. We aimed to decrease the cardiac oxygen requirement by starting levosimendan and decline afterload. If the patient, whose coronary artery disorder is detected, is under risk and his overall condition is bad, we think that coronary bypass operation can be delayed.
\end{abstract}

Keywords: Abdominal Aortic Aneurysm, Coronary Artery Disease, Levosimendan

\section{Introduction}

Abdominal aortic aneurysm (AAA) forms the most common type of aneurismal diseases. Generally, it is asymptomatic and when it is ruptured, it develops with high morbidity and mortality. While atherosclerosis generates the most frequent reason; smoking, male sex, hypertension, hyperlipidemia and collagen tissue disorder are the other risk factors.

\section{Case Report}

A 62-years-old male patient applied to our emergency with a pain beginning from the chest and spreading to the lumbar region. The patient's blood pressure was 55 $\mathrm{mmHg}-30 \mathrm{mmHg}$. With the support of intravenous solution, his blood pressure was kept under acceptable limits. With a suspicion of coronary artery disorder dissection, coronary angiography was executed. (Figure 1) Consecutive lesions of LAD artery (left anterior descending ) $40 \%-50 \%$ and $90 \%$, CX artery (circumflex) $40 \%$ and $80 \%-90 \%$, and a lesion of RCA (right coronary artery) $20 \%$ - 30\% were detected. Coronary angiography and then descendan, abdominal aortography were performed with the suspicion of dissection; by the way, abdominal aorta aneurysm was detected. With a suspicion of rupture, abdominal ultrasound was applied under emergency conditions. An aneurysm was detected 


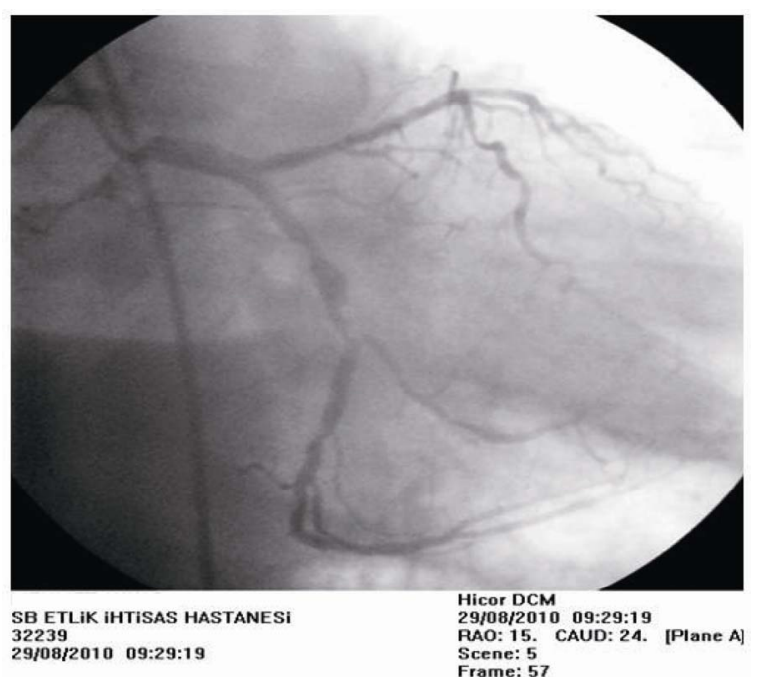

Figure 1. Coronary artery disease.

in USG and after the detection of flab in the aneurysm cyst, with a suspicion of dissection, contrasty thoracoabdominal tomography (CT) was demanded. In the tomography, a 7-cm-diameter ruptured abdominal aortic aneurysm was diagnosed. (Figure 2) In retroperitoneal region, a $23 \times 10 \times 7$-cm localized hematoma on the left kidney was detected. The patient was taken to the intensive care unit of cardiothoracic surgery. Levosimendan infusion was started with the lowest dosage due to low blood pressure. Chest pain was relieved after the infusion. The patient was stabilized in terms of coronary artery disease. His blood levels were Hgb 10 gr/dl, Hct 30\%, KB 70/30 mm Hg, pulse rate was 60/sec. He was operated under emergency conditions. Under the support of levosimendan, abdomen was opened with median laparotomy and it was detected that the aneurysm was ruptured from left back side. A Y graft operation was carried out from aorta to the bilateral iliac arteries by implementing a surgical clamp from the region below the kidney to the aorta. Excessive amount of hematoma was cleared from the outer surface of the left kidney and retroperitoneally. Because the patient's overall condition was deleterious and his hemodynamia wasn't stable, the operation was ended up with levosimendan support considering that coronary bypass would increase mortality and morbidity. Because heparin usage was increased the operation time. He didn't experience hemodynamic problem during his follow. 5 days after his operation, a pneumonia infection was added. The patient whose overall condition improved thanks to the suggestions of thoracic medicine and infection was discharged healthily 18 days after the operation.

\section{Discussion}

Abdominal aortic aneurysm is a clinical condition which

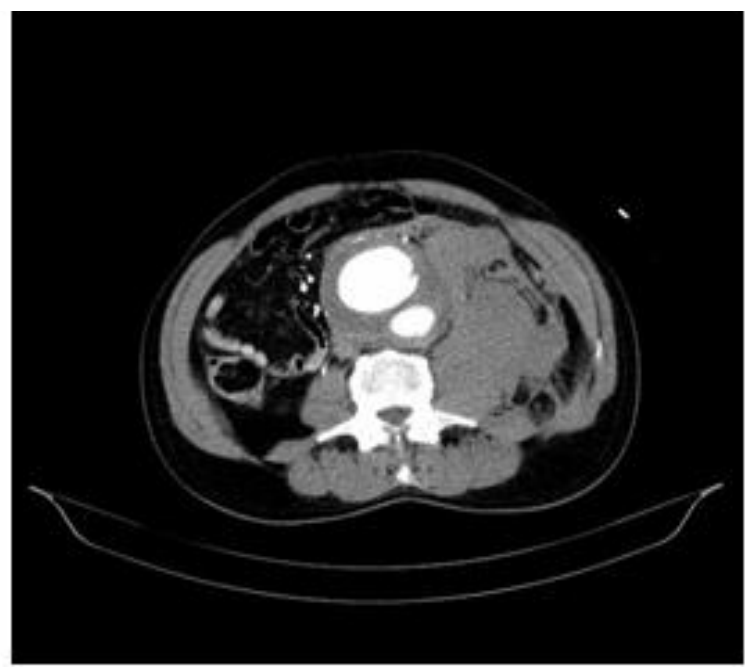

Figure 2. Ruptured abdominal aortic aneurysm.

improves silently and develops with high mortality when ruptured. Approximately $50 \%$ of the ruptured aneurysms are died before they reach hospital while the $30 \%-70 \%$ operated ones are died within 30 days after operation [1]. The mortality of aneurysms applied elective surgery ranges between $2 \%$ and $6 \%$ [1]. Many studies have indicated a significant unity between aneurysm and atherosclerosis [2-4]. Male patients, over the age 75 , have the risk of developing AAA 8 times more than female patients; in addition, smoking, the use of statin, male sex, high serum total cholesterol and lower HDL cholesterol increase the risk [1]. AAA can be diagnosed with the help of physical examination, ultrasound and tomogramphy. In 3\% of the cases, diagnosis cannot be achieved with ultrasound. Early diagnosis and follow is extremely important to decrease morbidity and mortality. After being ruptured, excessive increase in morbidity and mortality appears. The patients consulting with rupture must be taken to operations in emergency. Most patients don't have the opportunity to have additional examinations like coronary angio or etc. What should be done if coronary arteries disorder is detected in the patient ruptured and bypass is necessary? It is obvious that the first intervention is obligatory to the ruptured AAA. However, after the intervention to the aneurysm, should coronary bypass be applied or should there be following? In our opinion, a decision must be made according to the patient's clinic. Does subsequent coronary bypass operation increase morbidity and mortality? The existence of cardiovascular disorders and depressed cardiac functions are substantially significant in determining mortality and morbidity [5]. The existence of coronary artery disorder is an important factor to determine preoperative mortality. If AAA, which cannot be ruptured, is enormous and symptomatic, its rehabilitation can be done concurrently 
with coronary bypass surgery [6]. If AAA is not enormous and symptomatic, it can be rehabilitated 2 - 4 weeks after the coronary bypass surgery [6]. When combined, aneurysm rehabilitation can be done with beating bypass or partial cardiopulmonary bypass. It is detected that bleeding increases within the ones who had partial cardiopulmonary bypass [6]. In the critical patients, if cardiac functions and the oxygen amount reaching the heart can be provided within the optimal conditions during and after the operation, mortality and morbidity decrease noticeably $[7,8]$. In our case, the patient has requiring coronary bypass surgery. So to prevent this problem we prefer to use levosimendan. So, we supplied cardiac stabilization by increasing oxygen presentation and decreasing after- and pre-load and oxygen consumption.

Levosimendan have cytoprotective vasodilator properties and treatment of non-compensated heart failure. Levosimendan enhances myocardial contractility, decreasing myocardial oxygen comsumption [9]. It binds to calcium saturated cardiac troponin C (cTnC), this effect of levosimendan was shown to be dependent on the concentration of intracellular ionized calcium (Enhanced myocardial contractility, no increased oxygen consumption) $[10,11]$. The second mechanism stimulates ATP-sensitive potassium channels. (Anti stunning effects, anti ischaemic effects) Positive inotropic agents, phosphodiesterase inhibitors and adrenergic agonists such as dobutamine, associated with increasing myocardial oxygen demand and the potential to induce myocardial ischaemia or malignant arrhythmias [12,13]. Levosimendan may also exert vasodilator effects on human coronary conductance and resistance arteries [14]. After the drug infusion, coronary artery diameter, velocity, and flow increase significantly [14]. The most commonly adverse effects associated with the use of levosimendan are hypotension, headache, dizziness and nausea.

We avoided to use heparin requiring procedures because of our belief that heparin usage increases the bleeding. That is why we are reluctant the coronary bypass surgery the aneurysm repair. As a result of our case we thought that while repairing the abdominal aortic aneurysm the other comorbidites must be treated with medically.

Our patient consulted us with a shock state and his overall condition was bad. We aimed at decreasing the cardiac oxygen requirement by starting levosimendan and declining afterload. We increased the cardiac supply by rising blood pressure a bit. Considering that an application of coronary bypass would increase morbidity and mortality, we decided to follow up. If the patient, that has coronary artery disorder, is under risk and his overall condition is bad, we think that coronary bypass operation can be delayed.

\section{References}

[1] S. H. Forshdahl, K. Singh, S. Solberg and B. K. Jacobsen, "Risk Factors for Abdominal Aortic Aneurysms a 7-Year Prospective Study: The Tromso Study, 1994-2001,” Circulation, Vol. 119, 2009, pp. 2202-2208. doi:10.1161/CIRCULATIONAHA.108.817619

[2] K. Singh, K. H. Bonaa, B. K. Jacobsen, L. Björk and S. Solberg, "Prevalence of and Risk Factors for Abdominal Aortic Aneurysms in a Population-Based Study: The Tromso Study," American Journal of Epidemiology, Vol. 154, No. 3, 2001, pp. 236-244. doi:10.1093/aje/154.3.236

[3] D. Reed, C. Reed, G. Stemmermann and T. Hayashi, "Are Aortıc Aneurysms Caused by Atherosclerosis?" Circulation, Vol. 85, 1992, pp. 205-211.

[4] A. J. Lee, F. G. Fowkes, M. N. Carson, G. C. Leng and P. L. Allan, "Smoking, Atherosclerosis and Risk of Abdominal Aortic Aneurysm," European Heart Journal, Vol. 18, No. 4, 1997, pp. 671-676.

[5] S. Giordano, F. Biancari, P. Loponen, J. Wistbacka and M. Luther, "Preoperative Haemodynamic Parameters and the Immediate Outcome after Open Repair of Ruptured Abdominal Aortic Aneurysms," Interactive Cardiovascular Thoracic Surgery, Vol. 9, 2009, pp. 491-493.

[6] T. Wolff, D. Baykut, H. R. Zerkowski, P. Stierli and L. Gürke, “Combined Abdominal Aortic Aneurysm Repair and Coronary Artery Bypass: Presentation of 13 Cases and Review of the Literature," Annals of Vascular Surgery, Vol. 20, No. 1, 2006, pp. 23-29. doi:10.1007/s10016-005-9324-9

[7] I. Kantonen, M. Lepantalo, J. P. Salenius, S. Matzke, M. Luther and K. Ylönen, "Mortality in Abdominal Aortic Aneurysm Surgery: The Effect of Hospital Volume, Patient Mix and Surgeon's Case Load,” European Journal of Vascular and Endovascular Surgery, Vol. 14, No. 5, 1997, pp. 375-379. doi:10.1016/S1078-5884(97)80287-0

[8] T. Juvonen, F. Biancari, J. Rimpilainen, V. Anttila, M. Pokela, V. Vainionpaa, P. Romsi and K. Kiviluoma, "Determinants of Mortality after Hypothermic Circulatory Arrest in a Chronic Porcine Model," European Journal of Cardio-Thoracic Surgery, Vol. 20, No. 4, 2001, pp. 803-810.

[9] J. Levijoki, P. Pollesello, J. Kaivola, C. Tilgmann, T. Sorsa, A. Annila, I. Kilpelainen and H. Haikala, "Further Evidence for the Cardiac Troponin C Mediated Calcium Sensitization by Levosimendan. Structure-Response and Binding Analysis with Analogs of Levosimendan,” Journal of Molecular and Cellular Cardiology, Vol. 32, No. 3, 2000, pp. 479-491. doi:10.1006/jmcc.1999.1093

[10] Z. Papp, K. Csapo, P. Pollesello, H. Haikala and I. Edes, "Pharmacological Mechanisms Contributing to the Clinical Efficacy of Levosimendan,” Cardiovasc Drug Review, Vol. 23, No. 1, 2005, pp. 71-98. doi:10.1111/j.1527-3466.2005.tb00158.x

[11] D. P. Figgit, P. S. Gillies and K. L. Goa, "Levosimendan,” Drugs, Vol. 61, 2001, pp. 613-627. doi:10.2165/00003495-200161050-00006 
[12] M. Bayram, L. De Luca, B. M. Massie and M. Gheorghiade, "Dobutamine Milrinone and Dopamine in Acute Heart Failure Syndromes: A Reassessment," American Journal of Cardiology, Vol. 96, No. 6, 2005, pp. 47-58. doi:10.1016/j.amjcard.2005.07.021

[13] L. D. Caldicott, K. Hawley, R. Heppel, P. A. Woodmansey and K. S. Channer, "Intravenous Enoximone or Dobutamine for Severe Heart Failure after Acute Miyocardial Infarction: A Randomized Double-Blind Trial,”
European Heart Journal, Vol. 14, No. 5, 1993, pp. 696-700. doi:10.1093/eurheartj/14.5.696

[14] A. D. Michaels, B. McKeown, M. Kostal, K. T. Vakharia, M. V. Jordan, I. L. Gerber, E. Foster and K. Chatterjee, "Effects of Intravenous Levosimendan on Human Coronary Vasomotor Regulation, Left Ventricular Wall Stress, and Myocardial Oxygen Uptake,” Circulation, Vol. 111, 2005, pp. 1504-1509. doi:10.1161/01.CIR.0000159252.82444.22 